Data in second audit period, before the fluid prescription power plan had been implemented, showed that 51\% of IV maintenance fluids were isotonic. This increased to $88 \%$, after the power plan was introduced.

Across both the second and third audit periods, there were 2 infants who developed hyponatraemia after receiving maintenance IV fluids. One was on a mixture of TPN and isotonic fluid, the other was on hypotonic fluids. There was only one incident of hypernatraemia (146), which occurred while on $10 \%$ dextrose infusion.

Conclusion The prescription of isotonic solutions has increased significantly since the EPR power plan for paediatric fluid prescriptions was introduced. This had a much greater impact than trying to encourage guideline adherence alone.

There is little published evidence on the safety of isotonic fluids in young infants. We have not observed any significant hypernatraemia since the increase in isotonic fluid usage.

\section{G130(P) EVALUATION OF A YEAR OF DEBRIEFS IN A TERTIARY CHILDREN'S HOSPITAL AS PART OF A NEW FRAMEWORK OF STAFF SUPPORT}

H Conniff, C Macaulay. Evelina London Children's Hospital, Guys and St Thomas' NHS FT, London, UK

\subsection{6/archdischild-2020-rcpch.103}

Aims After a series of difficult resuscitations and serious incidents, we developed a new debrief pathway as part of a broader staff support programme. ${ }^{1}$ We report 12 month evaluation of this pathway.

Methods The debrief pathway includes: an immediate huddle after the event, a personal 'check in' within 48 hrs with a supervisor/line manager, and a debrief facilitated by a senior psychologist 1-3 weeks later. 71 debriefs were held over a year (52 group, 19 individual), the majority (67) delivered by the same psychologist. After each debrief, participants completed a semi-structured questionnaire and the Miller (2007) group outcomes scales.

Results Feedback from over 200 participants representing the full range of the MDT was collated. The overwhelming majority valued debriefs as demonstrated by the percentage of high scores (scoring 8, 9 or 10 on a scale $0-10$ ) on Miller group outcomes: $90 \%$ rated debriefs overall highly; 86\%, 89\% and 95\% rated highly respectively on Goals, Approach and Relationship with group/facilitator. If the immediate huddle and 48 hour follow up occurred, the debrief flowed more smoothly. Additionally, attendees appeared better adjusted and there were fewer requests for individual support.

Themes that emerged from the qualitative feedback include the importance of talking in a safe space, listening to different perspectives and that sharing emotional reactions improved their psychological wellbeing. Other themes include that debriefs help staff to recognise existing support and consider offering support to colleagues. Many staff reported debriefs had helped them remain in work or return to work after incidents. The analysis identified 'the protective power of staff support' - knowing that management sanctioned debriefs made people feel cared for by the hospital, and that debriefs improve working practice by providing the space to reflect on situations.

Conclusion Staff value debriefs following incidents. We found a staged approach to holding debriefs useful. This fits with a recent review reporting significant benefits from early trauma interventions when part of a wider package of support (Richins et al., 2019). We conclude that our debrief pathway, where debriefs are normalised and routine, plays an important role in the psychological wellbeing of our staff.

\section{REFERENCE}

1. The development of the staff support programme is discussed in more detail in another presentation.

\section{G131(P) THE ROLE OF VIRTUAL CLINICS IN REDUCING DEMAND FOR CLINIC CAPACITY- A PILOT STUDY FOR ANTENATALLY DETECTED HYDRONEPHROSIS PATHWAY AT A DISTRICT GENERAL HOSPITAL}

D Furnell, K Jada. Paediatrics, Royal Free London NHS FT, London, UK

\subsection{6/archdischild-2020-rcpch.104}

Aims To evaluate the use of telephone consultation 'virtual clinics' as an alternative to traditional face-to-face clinic in effectively managing the increasing demand for outpatient clinics. A pilot for antenatally detected hydronephrosis follow up service.

Methods Data from renal clinics at a district general hospital over one year was reviewed and information collected from patient electronic records.

Results There were 232 renal clinical appointments. 140/232 appointments were face-to-face (60\%) and 92/232 appointments were virtual (40\%). The DNA rate for face-to-face encounters was $10 / 140(7 \%)$ and for virtual clinics (no answer to telephone calls) was 4/93(4\%). Antenatal hydronephrosis related referrals accounted for 85/232 appointments (36.6\%), of which 38 were new referrals. 57/85 of all appointments for antenatal hydronephrosis were virtual $(67 \%)$ and 28 were face-to-face (33\%). The first appointment for all 38 of these patients was virtual (100\%). 5/38 patients were referred to tertiary services (13\%). 13/38 patients were discharged after the 1 st contact (34\%). Of those that needed further local follow up, $16 / 20$ patients were followed up by virtual clinic (64\%). Of the 16 followed up virtually only $2 / 16$ went on to require face-toface clinic at a later date (12\%).

Conclusion The incidence of antenatal hydronephrosis is around 1:200 with 50\% normalizing on postnatal scans. This patient cohort places considerable demand on outpatient capacity and parental and clinician time for scans and appointments. Antenatal hydronephrosis related appointments accounted for over a third of all renal clinic appointments. The use of virtual clinic has significantly reduced the number of face-to-face clinic appointments needed. Only $12 \%$ of patients referred went on to require face-to-face appointments, potentially freeing up outpatient clinic capacity and clinical time. It also has implications for parents in terms of visits for appointments and time off work. The DNA rates were better for virtual clinics than face-to-face appointments (4\% vs7\%). Transforming the way that outpatient consultations are delivered can improve patient and clinician experience, as well as allowing better management and reduction in demand. It may also offer significant opportunities for making financial savings for organisations. 